\title{
Clinical and radiological outcomes of chronic severe slipped capital femoral epiphysis patients treated by surgical dislocation and modified Dunn osteotomy: Case series
}

\author{
Sertan Hancıoğlu, MD1ㅁ, Hakan Koray Tosyalı, MD²D, Serkan Erkan, MD²(D, \\ Hüseyin Serhat Yercan, MD $^{2}$ (I) \\ ${ }^{1}$ Department of Orthopedics and Traumatology, Izmir University of Health Sciences, Tepecik Training and Research Hospital, Izmir, Turkey \\ ${ }^{2}$ Department of Orthopedics and Traumatology, Celal Bayar University, Faculty of Medicine, Manisa, Turkey
}

Slipped capital femoral epiphysis (SCFE) is the gradual posterior translation of the proximal femoral epiphysis from metaphysis. The etiology of SCFE remains mostly unclear. The most convenient treatment modality is controversial among orthopedic surgeons. In-situ fixation is the most preferred treatment option for stable mild or moderate slips, which stabilizes the epiphysis, while not correcting the residual deformity. The resultant deformity may lead to a loss of range of motion (ROM), femoroacetabular impingement (FAI), and later on to early osteoarthritis of the affected hip. Since the tendency to develop these mentioned complications is much higher in patients with chronic severe SCFE, attempts to obtain anatomical

Received: March 31, 2020

Accepted: May 06, 2020

Published online: September 11, 2020

Correspondence: Sertan Hancıoğlu, MD. İzmir SBÜ Tepecik Eğitim ve Araştırma Hastanesi, Ortopedi ve Travmatoloji Kliniği 35170 Yenişehir, Konak, İzmir, Türkiye.

E-mail: sertanh@hotmail.com

Doi: $10.5606 /$ ehc. 2020.75101

Citation: Hancıoğlu S, Tosyalı HK, Erkan S, Yercan HS. Clinical and radiological outcomes of chronic severe slipped capital femoral epiphysis patients treated by surgical dislocation and modified Dunn osteotomy: Case Series. Jt Dis Relat Surg 2020;31(3):599-604.

(2020 All right reserved by the Turkish Joint Diseases Foundation

This is an open access article under the terms of the Creative Commons Attribution-NonCommercial License, which permits use, distribution and reproduction in any medium, provided the original work is properly cited and is not used for commercial purposes (http://creativecommons.org/licenses/by-nc/4.0/)

\section{ABSTRACT}

Objectives: This study aims to evaluate the clinical and radiological outcomes of patients with chronic severe slipped capital femoral epiphysis (SCFE) treated by surgical dislocation and modified Dunn osteotomy (MDO).

Patients and methods: This retrospective study, conducted between January 2010 and December 2017, included nine patients ( 8 males, 1 female; mean age 12.6 years; range, 7 to 16 years) with nine hips. The degree of corrections in Southwick angle and alpha angle were measured on frog-leg views. Range of motion (ROM) of the hips was measured at each follow-up visit. Heyman and Herndon classification system and Harris Hip Score (HHS) were evaluated for clinical and functional outcomes at the latest follow-up visit.

Results: The mean follow-up time was 34.8 months. Avascular necrosis complication was observed in one hip. Except two patients with lateral femoral cutaneous neuropathy, none of the patients suffered from any other complications. Mean preoperative Southwick angle of $59.1^{\circ}$ was corrected to an angle of $-0.8^{\circ}$ postoperatively. Alpha angles were calculated as $44.6^{\circ}$ postoperatively. Modified Dunn osteotomy resulted in marked improvement in hip ROM in all directions and increased HHS.

Conclusion: Our results encourage us to use this method in treating SCFE patients with chronic severe slips.

Keywords: Modified Dunn osteotomy procedure, slipped capital femoral epiphysis, surgical hip dislocation.

reduction of the epiphysis can result in unacceptable complications, such as avascular necrosis (AVN), due to strectching of the posterior retinaculum including retinacular vessels. To overcome this devastating condition, Dunn ${ }^{[1]}$ proposed a technique of subcapital reorientation with trochanteric osteotomy. Ziebarth et al. ${ }^{[2]}$ modified this method by adding surgical dislocation described by Ganz et al., ${ }^{[3]}$ which facilitated 
direct observation of vascular flow of the epiphysis. This technique allowed anatomical reduction of the head without compromising vascular perfusion and finally reduced the risks for possible deformities of femoral head. In this study, we aimed to evaluate the clinical and radiological outcomes of patients with chronic severe SCFE treated by surgical dislocation and modified Dunn osteotomy (MDO).

\section{PATIENTS AND METHODS}

In this retrospective study conducted at Department of Orthopedics and Traumatology of Manisa Celal Bayar University Faculty of Medicine Hospital, we investigated the medical records of patients who had undergone MDO between January 2010 and December 2017. Patients with a diagnosis of chronic SCFE classified as either stable or instable SCFE according to Loder et al., ${ }^{[4]}$ with Southwick ${ }^{[5]}$ angle of $\geq 50^{\circ}$, and follow-up time of at least one year were included. Patients having other deformities or prior surgeries in other institutions were excluded. Totally nine patients ( 8 males, 1 female; mean age 12.6 years; range, 7 to 16 years) met the inclusion criteria. The study protocol was approved by the Izmir University of Health Sciences, Tepecik Training and Research Hospital Ethics Committee (No: 2019/16-32; 13/11/2019). A written informed consent was obtained from each patient. The study was conducted in accordance with the principles of the Declaration of Helsinki.

Hospital records were evaluated for history of symptoms, preoperative physical examinations, radiographs, and operative records. In order to obtain accurate measurements, all pre- and postoperative pelvis anteroposterior (AP) and frog-leg radiographs were uploaded to Probel PACS DICOM Viewer version 2.1.10 (Probel Yazılım, Izmir, Turkey). The degrees of correction in Southwick angle and alpha angle were measured on frog-leg views. ${ }^{[5]}$ Patients were recalled for follow-up at six weeks, three months, six months, one year, and annually. At each follow-up, physical examination was performed to assess the ROM and control radiographs were taken as well (Figure 1). Any progress to AVN of the femoral head, osteoarthritis, or FAI was noted. For clinical and functional outcomes at the latest follow-up, Heyman and Herndon classification system and Harris Hip Score (HHS) were used. ${ }^{[6,7]}$

All surgical interventions were performed by the same surgeon. Before the surgery, all patients received a single dose of cefazolin, calculated based on patient's weight. Under general anesthesia, the patients were placed in lateral decubitus position.
A longitudinal lateral incision was performed over the greater trochanter. Fascia lata was split in the line with incision. After trochanteric flip osteotomy, Z-shaped incision was performed over the capsule and surgical safe dislocation of the hip was established after excision of the ligamentum teres. Perfusion of the head was determined by drilling of the epiphysis with $1.5 \mathrm{~mm}$ Kirschner (K)-wires (Figure 1). After dislocation of the hip, the ipsilateral leg was placed in a sterile bag. Labral and cartilage damages were noted. Periosteum with its retinacular vessels was released from the femoral neck in order to preserve vascular perfusion of the head by avoiding stretching, which can occur after realignment of the slipped capital epiphysis (Figure 2). Posterolateral periosteum was released from the femoral neck with the remaining part of the greater trochanter. Anterior portion of the physis was identified and femoral head was gently separated from the femoral neck. Physis was removed by use of small curettes. After separation of the head from
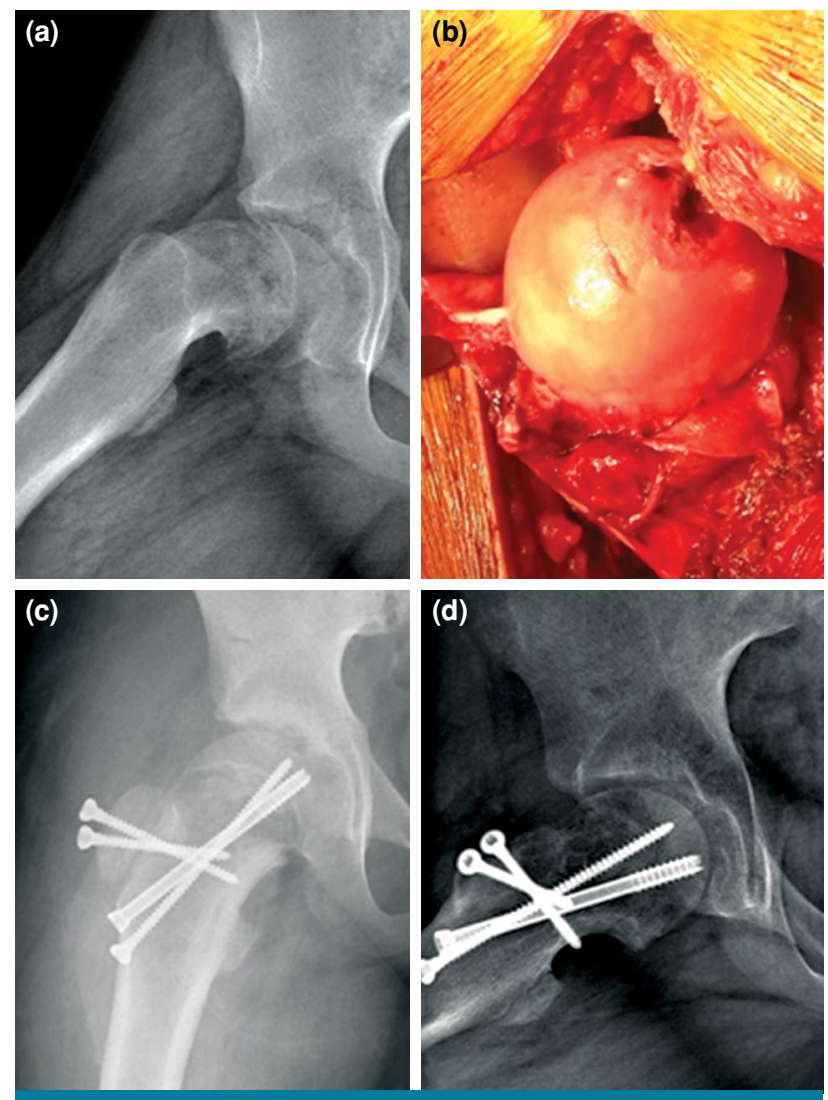

FIGURE 1. An 11-year-old female patient with right-sided slipped capital femoral epiphysis. (a) Preoperative slip angle was 58.50. (b) Intraoperative bleeding of femoral head after drilling. (c) Postoperative anteroposterior view. (d) First year follow-up, frog-leg lateral view with full range of motion. 


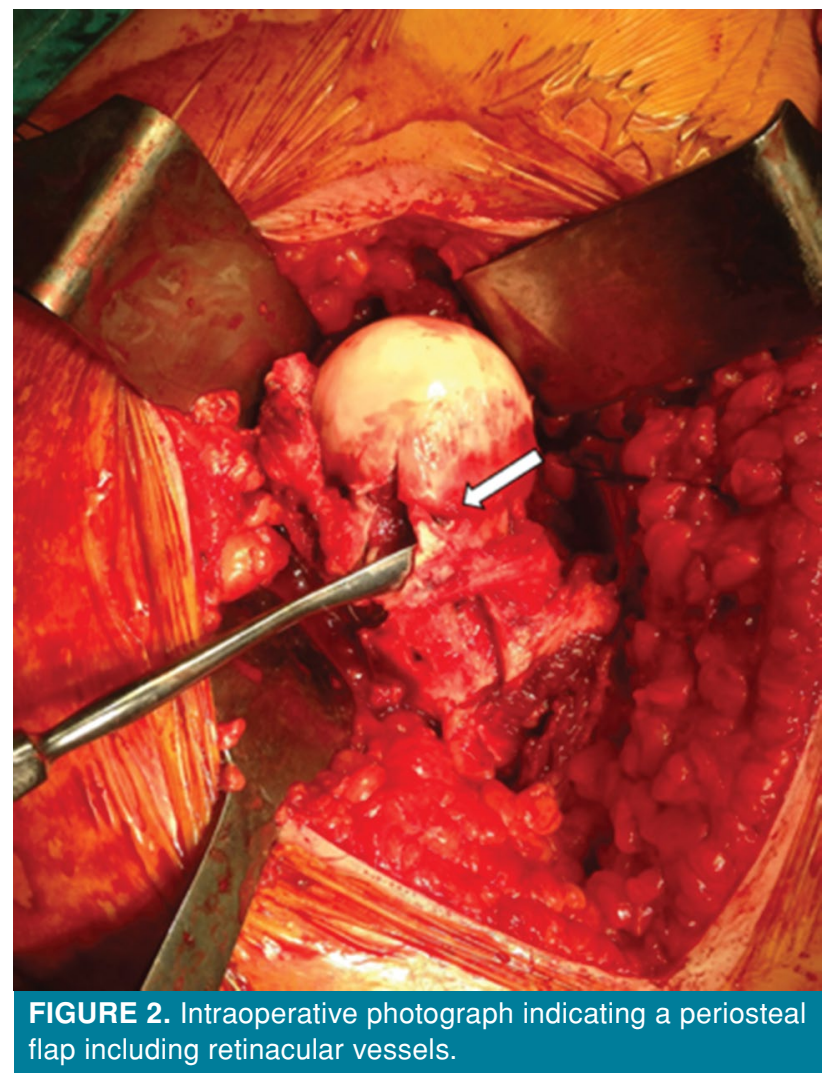

the neck, residual dorsal metaphyseal callus was removed by using appropriate bone chisels. The femoral head was reduced manually to the prepared neck in appropriate position and fixated with either $3 \mathrm{~mm}$ K-wires or cannulated/non-cannulated screws. After stable fixation, perfusion of the head was visualized again. Hip joint was gently reduced. Greater trochanter was replaced and fixed with two screws. After completion of the surgery, AP and frog-leg views were evaluated under fluoroscopic control.

Following surgery, antibiotic prophylaxis was administered to all patients for 48 hours. The same rehabilitation protocol was applied to all patients. Right after second day of surgery, patients were ambulated with touchdown weight bearing using two crutches. Postoperatively, active hip abduction was not allowed for four weeks in order to keep the fixation of greater trochanter safe. Passive flexion of hip over $90^{\circ}$ was not allowed until the bony union of head and neck was achieved. Afterwards, patients were encouraged for active flexion. After sixth week of postoperative period, patients were allowed to increase their weight-bearing status and they started full weight-bearing at second month.

\section{Statistical analysis}

Statistical analysis was performed by using IBM SPSS version 22.0 software (IBM Corp., Armonk, NY, USA). All statistical data were described as distribution and mean values. Shapiro-Wilk test showed that the parameters were not distributed normally. Non-parametric Wilcoxon signed-rank test was used to statistically assess the non-parametric values. Radiological results, ROM, and HHS in the pre- and postoperative periods were compared in pairwise manner. The statistical data were accepted as significant if the $\mathrm{p}<0.05$.

\section{RESULTS}

The mean follow-up time was 34.8 (range, 12 to 77 ) months. Eight hips were chronic and one hip was acute-on-chronic. Two patients were classified as unstable according to Loder (Table I). Drehmann sign was positive in all cases. One patient with bilateral SCFE (left hip was severe) had a prior in-situ fixation history for both hips in our clinic. After the fixation failure of the left severe hip, patient had undergone a revision surgery with implant removal and MDO.

Intraoperative findings revealed that all hips had separation of anterior periosteum from femoral neck and extensive synovitis in the acetabular fossa. Anterior labral fraying was detected in all cases. Labral tear was described in two patients. Epiphyseal perfusion was normal with active bleeding both before and after reduction of the hip during intraoperative evaluation.

\begin{tabular}{|c|c|c|c|}
\hline \multicolumn{4}{|c|}{$\begin{array}{c}\text { TABLE I } \\
\text { Patient's demographic characteristics }\end{array}$} \\
\hline & $\mathrm{n}$ & Mean & Range \\
\hline Age (year) & & 12.6 & $7-16$ \\
\hline \multicolumn{4}{|l|}{ Sex } \\
\hline Male & 8 & & \\
\hline Female & 1 & & \\
\hline \multicolumn{4}{|l|}{ Side } \\
\hline Right & 5 & & \\
\hline Left & 4 & & \\
\hline Follow-up time (month) & & 34.8 & $12-77$ \\
\hline \multicolumn{4}{|l|}{ Chronicity } \\
\hline Chronic & 8 & & \\
\hline Acute on chronic & 1 & & \\
\hline \multicolumn{4}{|l|}{ Loder classification } \\
\hline Stable & 7 & & \\
\hline Unstable & 2 & & \\
\hline
\end{tabular}




\begin{tabular}{|c|c|c|c|}
\hline & $\begin{array}{l}\text { TABLE I } \\
\text { ladiographic fi }\end{array}$ & dings & \\
\hline & Preoperative & Postoperative & \\
\hline & Mean $\pm S D$ & Mean \pm SD & $p$ \\
\hline Southwick angle & $59.1 \pm 10.6^{\circ}$ & $-0.8 \pm 0.7^{\circ}$ & 0.004 \\
\hline Alpha angle & $103.3 \pm 32.7^{\circ}$ & $44.6 \pm 9.1^{\circ}$ & 0.004 \\
\hline
\end{tabular}

\begin{tabular}{|c|c|c|c|}
\hline \multicolumn{4}{|c|}{$\begin{array}{c}\text { TABLE III } \\
\text { Clinical findings }\end{array}$} \\
\hline & Preoperative & Postoperative & \\
\hline & Mean \pm SD & Mean $\pm S D$ & $p$ \\
\hline \multicolumn{4}{|c|}{ Range of motion } \\
\hline IR & $0 \pm 0^{\circ}$ & $28.3 \pm 9.4^{\circ}$ & 0.004 \\
\hline Flexion & $85 \pm 3.6^{\circ}$ & $105.6 \pm 10.4^{\circ}$ & 0.004 \\
\hline ER & $18.3 \pm 4.3^{\circ}$ & $46.1 \pm 5.5^{\circ}$ & 0.004 \\
\hline Extension & $2.8 \pm 2^{\circ}$ & $5.1 \pm 2.4^{\circ}$ & 0.016 \\
\hline Abduction & $15.6 \pm 3.9^{\circ}$ & $40 \pm 7.5^{\circ}$ & 0.004 \\
\hline HHS & $63.2 \pm 3.6^{\circ}$ & $94.2 \pm 11.3^{\circ}$ & 0.004 \\
\hline
\end{tabular}

SD: Standard deviation; IR: Internal rotation; ER: External rotation; HHS: Harris Hip Score.

Avascular necrosis was observed in one hip at eighth postoperative month. None of the patients suffered from any other complications such as nerve injuries, deep venous thrombosis, heterotrophic ossification or infection except two patients with lateral femoral cutaneous neuropathy, which was resolved completely after one year of surgery.

Mean preoperative Southwick angle was $59.1^{\circ}$ (range, $50.6^{\circ}$ to $75^{\circ}$ ) and improved significantly to mean $-0.8^{\circ}$ (range, $-1.2^{\circ}$ to $1.32^{\circ}$ ) postoperatively with MDO technique $(\mathrm{p}=0.004)$. In comparison to preoperative values, alpha angles demonstrated a significant correction and were measured as a mean $44.6^{\circ}$ (range, $32^{\circ}$ to $62.1^{\circ}$ ) postoperatively $(\mathrm{p}=0.004$ ) (Table II).

The MDO resulted in significant improvement in hip ROM in all directions $(\mathrm{p}<0.05)$. Follow-up functional results yielded significant improvement. HHS increased from mean $63.2 \pm 3.6)$ preoperatively to $94.2 \pm 11.3)$ postoperatively $(\mathrm{p}=0.004)$. These results are summarized in Table III. Seven patients had excellent and one patient had good outcomes according to Heyman and Herndon classification at latest followup, whereas one patient, who suffered from AVN, had a poor result. The patient with AVN underwent implant removal and hip arthrodesis by using the cobra head plate.

\section{DISCUSSION}

There are a limited number of studies about the effect of MDO on severe SCFE and recent studies mostly compare it with in-situ fixation. Although in-situ fixation is the most commonly used technique in treating SCFE, this technique is mostly inadequate to avoid FAI, even in mild slips. ${ }^{[8]}$ In our clinic, in-situ fixation technique is the only preferred technique in treating patients having mild or moderate slips, and $\mathrm{MDO}$ is performed only in severe slips. Therefore, any comparison between in-situ fixation and MDO was not rational to be assessed in our study. ${ }^{[0]}$

In severe SCFE, metaphysis becomes more prominent on epiphysis, which impact on acetabular rim particularly when the hip is flexed. This impingement leads to damaging forces on labrum and causes injuries on cartilage of femoral head. ${ }^{[10]}$ The realignment procedure of modified Dunn offers a near anatomical correction of the distorted alignment of femoral head and neck by using safe surgical dislocation of the hip. This intervention does not affect the vascular blood supply of the femoral head and therefore decreases the risk of osteonecrosis. ${ }^{[2,11]}$

The number of patients with severe SCFE treated with MDO technique in the literature was comparable to our study. Elmarghany et al. ${ }^{[12]}$ investigated the results of 32 patients treated with MDO and 20 of the patients had severe SCFE. Slongo et al. ${ }^{[13]}$ reported the results of 23 patients with SCFE treated with MDO and there were 14 patients with mild and moderate and nine patients with severe slips. Several studies compared the results of MDO technique with in-situ pinning in patients with SCFE. Novais et al. ${ }^{[14]}$ compared patients only with severe SCFE treated either by in-situ pinning or MDO technique, where MDO procedure was performed in 15 patients. In the comparative study by Cosma et al., ${ }^{[15]}$ in-situ pinning and MDO procedure were compared by means of their femoral neck shortening, where 10 patients with in-situ fixation had varying degrees of slip angle and seven patients treated with MDO had only severe slips similar to our study. Guindani et al. ${ }^{[16]}$ conducted a single-center study and evaluated the threeyear follow-up clinical and radiological outcomes of 23 hips with severe SCFE treated with MDO technique. Moreover, Trisolino et al. ${ }^{[17]}$ compared the two techniques only in severe slips and the number of patients treated with MDO was 15 . With a large sample size of 28 patients, Madan et al. ${ }^{[18]}$ investigated the results of MDO technique in severe slips including acute cases.

In our study, the mean preoperative Southwick angle was $59.1^{\circ}$ (range, $50.6^{\circ}$ to $75^{\circ}$ ). This finding was 
comparable with the results of other studies: Novais et al. ${ }^{[14]}$ observed the aforementioned angle as $65^{\circ}$ (range, $54^{\circ}$ to $81^{\circ}$ ), Trisolino et al. ${ }^{[17]}$ calculated it as $68^{\circ} \pm 11^{\circ}$, and Guindani et al..$^{[16]}$ found it as $70^{\circ}$ (range, $60^{\circ}$ to $90^{\circ}$ ). In the current study, we demonstrated that the mean postoperative Southwick angle improved to $-0.8^{\circ}$. This improvement was comparable to the findings of Slongo et al. ${ }^{[13]}$ and Cosma et al. ${ }^{[15]}$ They observed the improvement of the mean Southwick angle from $47.6^{\circ}$ to $4.6^{\circ}$ and from $68^{\circ}$ to $9^{\circ}$, respectively. Madan et al..$^{[18]}$ corrected the lateral slip angle from $59^{\circ} \pm 11.6^{\circ}$ to $8^{\circ} \pm 7.9^{\circ}$ as well.

We observed that the mean alpha angle was corrected from $103.3^{\circ}$ preoperatively to $44.6^{\circ}$ postoperatively. This amount of correction was comparable to the results of other studies in the literature. Elmarghany et al. ${ }^{[12]}$ revealed a correction in alpha angle from $97.85^{\circ}$ to $51.15^{\circ}$ postoperatively. Similar results were reported in the study of Novais et al. ${ }^{[14]}$ Guindani et al. ${ }^{[16]}$ calculated postoperative alpha angle as $60^{\circ}$. In the comparative study of Trisolino et al., ${ }^{[17]}$ it was stated that patients treated with MDO procedure had a mean alpha angle of $50^{\circ}$ whereas patients treated with in-situ fixation had a mean alpha angle of $89^{\circ}$ postoperatively.

We detected that the MDO procedure resulted in significant improvement in ROM and functional scores in postoperative period. In comparison to in-situ fixation, Novais et al. ${ }^{[14]}$ reported that patients treated with MDO procedure yielded better results in Heyman and Herndon clinical classification, where nine out of 15 patients had good and excellent scores. In our study, postoperative HHS was improved to 94.2. This finding was compatible with the results of the other studies in the literature. Massè et al. ${ }^{[19]}$ reported that the mean HHS was evaluated as 98.2 postoperatively. Guindani et al. ${ }^{[16]}$ reported the mean postoperative HHS as 91.2 and Madan et al. ${ }^{[18]}$ demonstrated an improvement in HHS from a mean value of 19.6 to 89.1 .

In the current study, one out of nine (11.1\%) patients suffered from AVN as a complication. Although the study's small sample size constituted a major limitation, the rate of complications was comparable to the results of previous studies. Cosma et al. ${ }^{[15]}$ reported that one out of seven (14.2\%) patients had AVN as a complication. Guindani et al. ${ }^{[16]}$ revealed that eight out of 23 patients had complications, where four $(17 \%)$ had AVN and implant failure, heterotrophic ossification while implant loosening constituted the rest of the complications. Trisolino et al. ${ }^{[17]}$ reported three cases with AVN out of 15 patients treated with MDO procedure and two of them underwent total hip replacement surgery in approximately 1.5 years. Madan et al. ${ }^{[18]}$ observed AVN in four patients out of 28 patients, and two of them had unstable slips and signs of AVN even in the preoperative period. In contrast to these findings, Ziebarth et al. ${ }^{[2]}$ reported that none of the 40 patients suffered from AVN; however, three had heterotrophic ossification, three had implant failure, three had delayed union, and one patient had FAI.

Although MDO is a long known procedure performed by experienced surgeons, there is a lack of algorithm in the planning of treatment, particularly in the selection of the proper patient. Alves et al. ${ }^{[20]}$ performed a study evaluating unstable cases, realized a rate of AVN as $67 \%$, and proposed that precise attention should be paid and adequate experience has to be gained in treating unstable cases. Persinger et al. ${ }^{[21]}$ treated 31 unstable hips with MDO procedure and $6 \%$ of the patients who suffered from AVN underwent surgery in 20 hours after admission. These patients were free of AVN after the operation.

The most important limitations of this study are the retrospective nature and the small sample size. Our study included patients with only severe SCFE whereas previous studies involved mild, moderate, and severe cases. This status improves the results of our study regarding the homogeneity of the study group.

In conclusion, although the number of patients in this study is limited, our results were compatible with the findings in the literature and encourage us to use this method in treating severe slips in a safe manner. In order to eliminate the small sample size and diversities of indications, one should design a prospective, multi-centric study and evaluate the long-term results of MDO procedure in treating patients with severe SCFE.

\section{Declaration of conflicting interests}

The authors declared no conflicts of interest with respect to the authorship and/or publication of this article.

\section{Funding}

The authors received no financial support for the research and/or authorship of this article.

\section{REFERENCES}

1. Dunn DM. The treatment of adolescent slipping of the upper femoral epiphysis. J Bone Joint Surg [Br] 1964;46:621-9.

2. Ziebarth K, Zilkens C, Spencer S, Leunig M, Ganz R, Kim YJ. Capital realignment for moderate and severe SCFE using a modified Dunn procedure. Clin Orthop Relat Res 2009;467:704-16. 
3. Ganz R, Gill TJ, Gautier E, Ganz K, Krügel N, Berlemann U. Surgical dislocation of the adult hip a technique with full access to the femoral head and acetabulum without the risk of avascular necrosis. J Bone Joint Surg [Br] 2001;83:1119-24.

4. Loder RT, Richards BS, Shapiro PS, Reznick LR, Aronson DD. Acute slipped capital femoral epiphysis: the importance of physeal stability. J Bone Joint Surg [Am] 1993;75:1134-40.

5. Southwick WO. Osteotomy through the lesser trochanter for slipped capital femoral epiphysis. J Bone Joint Surg [Am] 1967;49:807-35.

6. Heyman $\mathrm{CH}$, Herndon $\mathrm{CH}$. Epiphy seodesis for early slipping of the upper femoral epiphysis. J Bone Joint Surg [Am] 1954;36-A:539-55.

7. Harris WH. Traumatic arthritis of the hip after dislocation and acetabular fractures: treatment by mold arthroplasty. An end-result study using a new method of result evaluation. J Bone Joint Surg [Am] 1969;51:737-55.

8. Millis MB, Novais EN. In situ fixation for slipped capital femoral epiphysis: perspectives in 2011. J Bone Joint Surg [Am] 2011;93 Suppl 2:46-51.

9. Atik OŞ. Is there something new and interesting in my article? Eklem Hastalik Cerrahisi 2019;30:69.

10. Leunig M, Casillas MM, Hamlet M, Hersche O, Nötzli H, Slongo $\mathrm{T}$, et al. Slipped capital femoral epiphysis: early mechanical damage to the acetabular cartilage by a prominent femoral metaphysis. Acta Orthop Scand 2000;71:370-5.

11. Huber H, Dora C, Ramseier LE, Buck F, Dierauer S. Adolescent slipped capital femoral epiphysis treated by a modified Dunn osteotomy with surgical hip dislocation. J Bone Joint Surg [Br] 2011;93:833-8.

12. Elmarghany M, Abd El-Ghaffar TM, Seddik M, Akar A, Gad Y, Ragheb E, et al. Surgical hip dislocation in treatment of slipped capital femoral epiphysis. SICOT J 2017;3:10.

13. Slongo T, Kakaty D, Krause F, Ziebarth K. Treatment of slipped capital femoral epiphysis with a modified Dunn procedure. J Bone Joint Surg [Am] 2010;92:2898-908.

14. Novais EN, Hill MK, Carry PM, Heare TC, Sink EL. Modified Dunn Procedure is Superior to In Situ Pinning for Short-term Clinical and Radiographic Improvement in Severe Stable SCFE. Clin Orthop Relat Res 2015;473:2108-17.

15. Cosma D, Vasilescu DE, Corbu A, Văleanu M, Vasilescu D. The modified Dunn procedure for slipped capital femoral epiphysis does not reduce the length of the femoral neck. Pak J Med Sci 2016;32:379-84.

16. Guindani N, Eberhardt O, Wirth T, Surace MF, Fernandez FF. The Modified Dunn Osteotomy for SCFE: Clinical and radiographical results at 3 years follow-up. J Hip Surg 2017;1:173-9.

17. Trisolino G, Stilli S, Gallone G, Santos Leite P, Pignatti G. Comparison between modified Dunn procedure and in situ fixation for severe stable slipped capital femoral epiphysis. Acta Orthop 2018;89:211-6.

18. Madan SS, Cooper AP, Davies AG, Fernandes JA. The treatment of severe slipped capital femoral epiphysis via the Ganz surgical dislocation and anatomical reduction: a prospective study. Bone Joint J 2013;95-B:424-9.

19. Massè A, Aprato A, Grappiolo G, Turchetto L, Campacci A, Ganz R. Surgical hip dislocation for anatomic reorientation of slipped capital femoral epiphysis: preliminary results. Hip Int 2012;22:137-44.

20. Alves C, Steele M, Narayanan U, Howard A, Alman B, Wright JG. Open reduction and internal fixation of unstable slipped capital femoral epiphysis by means of surgical dislocation does not decrease the rate of avascular necrosis: a preliminary study. J Child Orthop 2012;6:277-83.

21. Persinger F, Davis RL 2nd, Samora WP, Klingele KE. Treatment of Unstable Slipped Capital Epiphysis Via the Modified Dunn Procedure. J Pediatr Orthop 2018;38:3-8. 